\title{
Further results on $A$-numerical radius inequalities
}

\author{
Nirmal Chandra Rout ${ }^{1} \cdot$ Debasisha Mishra $^{1}$ (D)
}

Received: 10 May 2021 / Accepted: 14 November 2021 / Published online: 6 December 2021

(c) Tusi Mathematical Research Group (TMRG) 2021

\begin{abstract}
Let $A$ be a bounded linear positive operator on a complex Hilbert space $\mathcal{H}$. Furthermore, let $\mathcal{B}_{A} \Leftarrow \mathcal{H} \Rightarrow$ denote the set of all bounded linear operators on $\mathcal{H}$ whose $A$-adjoint exists, and $\mathbb{A}$ signify a diagonal operator matrix with diagonal entries are $A$. Very recently, several $\mathbb{A}$-numerical radius inequalities of $2 \times 2$ operator matrices were established. In this paper, we prove a few new $\mathbb{A}$-numerical radius inequalities for $2 \times 2$ and $n \times n$ operator matrices. We also provide a new proof of an existing result by relaxing a sufficient condition " $A$ is strictly positive". Our proofs show the importance of the theory of the Moore-Penrose inverse of a bounded linear operator in this field of study.
\end{abstract}

Keywords A-numerical radius $\cdot$ Moore-Penrose inverse $\cdot$ Positive operator $\cdot$ Semiinner product $\cdot$ Inequality $\cdot$ Operator matrix

Mathematics Subject Classification 47A12 - 47A30 - 47A63 - 47A05

\section{Introduction}

Throughout, $\mathcal{H}$ denotes a complex Hilbert space with inner product $\langle\cdot, \cdot\rangle$. By $\mathcal{B}(\mathcal{H})$, we mean the $C^{*}$-algebra of all bounded linear operators on $\mathcal{H}$. Let $\|\cdot\|$ be the norm induced from $\langle\cdot, \cdot\rangle$. For $A \in \mathcal{B}(\mathcal{H}), \mathcal{R}(A)$ stands for the range space of $A$ and $\overline{\mathcal{R}(A)}$ for the norm closure of $\mathcal{R}(A)$ in $\mathcal{H}$. And $A^{*}$ represents the adjoint operator of $A$. An operator $A \in \mathcal{B}(\mathcal{H})$ is called selfadjoint if $A=A^{*}$. A selfadjoint operator $A \in \mathcal{B}(\mathcal{H})$ is called positive if $\langle A x, x\rangle \geq 0$ for all $x \in \mathcal{H}$, and is called strictly positive if $\langle A x, x\rangle>0$ for all non-zero $x \in \mathcal{H}$. If $A$ is a positive (strictly positive)

Communicated by Qing-Wen Wang.

Debasisha Mishra

kapamath@gmail.com

Nirmal Chandra Rout

nrout89@gmail.com

1 Department of Mathematics, National Institute of Technology Raipur, Raipur 492010, India 
operator, then we use the notation $A \geq 0(A>0)$. Let $\mathbb{A}$ be an $n \times n$ diagonal operator matrix whose diagonal entries are positive operator $A$ for $n=1,2, \ldots$. Then, $\mathbb{A} \in \mathcal{B}\left(\bigoplus_{i=1}^{n} \mathcal{H}\right)$ and $\mathbb{A} \geq 0$. If $A \geq 0$, then it induces a positive semidefinite sesquilinear form, $\langle\cdot, \cdot\rangle_{A}: \mathcal{H} \times \mathcal{H} \rightarrow \mathbb{C}$ defined by $\langle x, y\rangle_{A}=\langle A x, y\rangle, x, y \in \mathcal{H}$. Let $\|\cdot\|_{A}$ denote the seminorm on $\mathcal{H}$ induced by $\langle\cdot, \cdot\rangle_{A}$, i.e., $\|x\|_{A}=\sqrt{\langle x, x\rangle_{A}}$ for all $x \in \mathcal{H}$. Then, $\|x\|_{A}$ is a norm if and only if $A>0$. Also, $\left(\mathcal{H},\|\cdot\|_{A}\right)$ is complete if and only if $\mathcal{R}(A)$ is closed in $\mathcal{H}$. Henceforth, we use the symbol $A$ and $\mathbb{A}$ for positive operators on $\mathcal{H}$ and $\bigoplus_{i=1}^{n} \mathcal{H}$, respectively. We retain the notations $O$ and $I$ for the null operator and the identity operator on $\mathcal{H}$, respectively. Given $T \in \mathcal{B} \Leftarrow \mathcal{H} \Rightarrow$, the $A$-operator seminorm $\|T\|_{A}$ is defined as follows:

$$
\|T\|_{A}=\sup _{x \in \overline{\mathcal{R}(A)}, x \neq 0} \frac{\|T x\|_{A}}{\|x\|_{A}}=\inf \left\{c>0:\|T x\|_{A} \leq c\|x\|_{A}, 0 \neq x \in \overline{\mathcal{R}(A)}\right\}<\infty .
$$

We set $\mathcal{B}^{A} \Leftarrow \mathcal{H} \Rightarrow=\left\{T \in \mathcal{B} \Leftarrow \mathcal{H} \Rightarrow:\|T\|_{A}<\infty\right\}$. Then $\mathcal{B}^{A} \Leftarrow \mathcal{H} \Rightarrow$ is not a subalgebra of $\mathcal{B} \Leftarrow \mathcal{H} \Rightarrow$. It is pertinent to point out that $\|T\|_{A}=0$ if and only if $A T A=O$. For $T \in \mathcal{B}^{A} \Leftarrow \mathcal{H} \Rightarrow$, we have

$$
\|T\|_{A}=\sup \left\{\left|\langle T x, y\rangle_{A}\right|: x, y \in \overline{\mathcal{R}(A)},\|x\|_{A}=\|y\|_{A}=1\right\} .
$$

If $A T \geq 0$, then the operator $T$ is called $A$-positive. Note that if $T$ is $A$-positive, then

$$
\|T\|_{A}=\sup \left\{\langle T x, x\rangle_{A}: x \in \mathcal{H},\|x\|_{A}=1\right\} .
$$

Before we proceed further, it is necessary to introduce the concept of $A$-adjoint operator. We say an operator $X \in \mathcal{B} \Leftarrow \mathcal{H} \Rightarrow$ to be $A$-adjoint operator of $T \in \mathcal{B} \Leftarrow \mathcal{H} \Rightarrow$ if $\langle T x, y\rangle_{A}=\langle x, X y\rangle_{A}$ for every $x, y \in \mathcal{H}$, i.e., $A X=T^{*} A$. By Douglas Theorem [5], the existence of an $A$-adjoint operator is not guaranteed. An operator $T \in \mathcal{B} \Leftarrow \mathcal{H} \Rightarrow$ may admit none, one or many $A$-adjoints. A rather well-known result states that $A$-adjoint of an operator $T \in \mathcal{B} \Leftarrow \mathcal{H} \Rightarrow$ exists if and only if $\mathcal{R}\left(T^{*} A\right) \subseteq \mathcal{R}(A)$. Let us now denote $\mathcal{B}_{A} \Leftarrow \mathcal{H} \Rightarrow=\left\{T \in \mathcal{B} \Leftarrow \mathcal{H} \Rightarrow: \mathcal{R}\left(T^{*} A\right) \subseteq \mathcal{R}(A)\right\}$. Note that $\mathcal{B}_{A} \Leftarrow \mathcal{H} \Rightarrow$ is a subalgebra of $\mathcal{B} \Leftarrow \mathcal{H} \Rightarrow$ which is neither closed nor dense in $\mathcal{B} \Leftarrow \mathcal{H} \Rightarrow$. Moreover, we have the following inclusion relations:

$$
\mathcal{B}_{A} \Leftarrow \mathcal{H} \Rightarrow \subseteq \mathcal{B}^{A} \Leftarrow \mathcal{H} \Rightarrow \subseteq \mathcal{B} \Leftarrow \mathcal{H} \Rightarrow
$$

And the equality holds if $A$ is injective and has a closed range.

For $T \in \mathcal{B} \Leftarrow \mathcal{H} \Rightarrow, w_{A}(T)$, the A-numerical radius of $T$ was proposed by Saddi [13]. And is defined as follows:

$$
w_{A}(T)=\sup \left\{\left|\langle T x, x\rangle_{A}\right|: x \in \mathcal{H},\|x\|_{A}=1\right\} .
$$

When $T=\left(T_{i j}\right)$ is an $n \times n$ operator matrix with $T_{i j} \in \mathcal{B} \Leftarrow \mathcal{H} \Rightarrow$, then (1) can be written as

$$
w_{\text {A }}(T)=\sup \left\{\left|\langle T x, x\rangle_{\mathbb{A}}\right|: x \in \bigoplus_{n=1}^{n} \mathcal{H},\|x\|_{\mathbb{A}}=1\right\} .
$$


Very recently, Zamani [16] obtained the following $A$-numerical radius inequality for $T \in \mathcal{B}_{A}(\mathcal{H})$ :

$$
\frac{1}{2}\|T\|_{A} \leq w_{A}(T) \leq\|T\|_{A} .
$$

The first inequality in (2) becomes an equality if $T^{2}=O$ and the second inequality becomes an equality if $T$ is $A$-selfadjoint. The $A$-Crawford number of $T \in \mathcal{B}_{A}(\mathcal{H})$ is defined as

$$
c_{A}(T)=\inf \left\{\left|\langle T x, x\rangle_{A}\right|: x \in \mathcal{H},\|x\|_{A}=1\right\} .
$$

This terminology was introduced by Zamani [16]. Furthermore, if $T$ is $A$-selfadjoint, then $w_{A}(T)=\|T\|_{A}$. Moslehian et al. [8] continued the study of $A$-numerical radius and obtained some new $A$-numerical radius inequalities. In this year, Bhunia et al. [3] presented several $\mathbb{A}$-numerical radius inequalities for a strictly positive operator $A$. We refer the interested reader to $[12,14-16]$ and the references cited therein for further generalizations and refinements of $A$-numerical radius inequalities.

The objective of this paper is to present a few new A-numerical radius inequalities for $2 \times 2$ and $n \times n$ operator matrices. Besides these, we aim to establish some existing A-numerical radius inequalities by relaxing sufficient condition $A>0$. To this end, the paper is sectioned as follows. In Sect. 2, we define additional mathematical constructs including the definition of the Moore-Penrose inverse of an operator, $A$-adjoint, $A$-selfadjoint and $A$-unitary operator, that are required to state and prove the results in the subsequent sections. Section 3 contains several new $A$-numerical radius inequalities. More interestingly, it also provides some recent existing results in the literature on $A$-numerical radius inequalities by dropping a sufficient condition.

\section{Preliminaries}

This section gathers a few more definitions and results that are useful in proving our main results. It starts with the definition of the Moore-Penrose inverse of a bounded operator $A$ in $\mathcal{H}$. The Moore-Penrose inverse of $A \in \mathcal{B} \Leftarrow \mathcal{H} \Rightarrow$ [9] is the operator $X: \mathcal{R}(A) \bigoplus \mathcal{R}(A)^{\perp} \longrightarrow \mathcal{H}$ which satisfies the following four equations:

$$
\begin{array}{r}
\text { (1) } A X A=A, \text { (2) } X A X=X, \text { (3) } X A=P_{\mathcal{N}(A)^{\perp}}, \text { (4) } \\
A X=\left.P_{\overline{\mathcal{R}(A)}}\right|_{\mathcal{R}(A) \oplus \mathcal{R}(A)^{\perp} .}
\end{array}
$$

Here, $\mathcal{N}(A)$ and $P_{L}$ denote the null space of $A$ and the orthogonal projection onto $L$, respectively. The Moore-Penrose inverse is unique, and is denoted by $A^{\dagger}$. In general, $A^{\dagger} \notin \mathcal{B} \Leftarrow \mathcal{H} \Rightarrow$. It is bounded if and only if $\mathcal{R}(A)$ is closed. If $A \in \mathcal{B} \Leftarrow \mathcal{H} \Rightarrow$ is invertible, then $A^{\dagger}=A^{-1}$. If $T \in \mathcal{B}_{A} \Leftarrow \mathcal{H} \Rightarrow$, the reduced solution of the equation $A X=T^{*} A$ is a distinguished $A$-adjoint operator of $T$, which is denoted by $T^{\#_{A}}$ (see $[2,7])$. Note that $T^{\#_{A}}=A^{\dagger} T^{*} A$. If $T \in \mathcal{B}_{A}(\mathcal{H})$, then $A T^{\#_{A}}=T^{*} A, \mathcal{R}\left(T^{\#_{A}}\right) \subseteq \overline{\mathcal{R}(A)}$ and $\mathcal{N}\left(T^{\#_{A}}\right)=\mathcal{N}\left(T^{*} A\right)($ see $[5])$. One can observe that 


$$
I^{\#_{A}}=A^{\dagger} I^{*} A=A^{\dagger} A=P_{\overline{\mathcal{R}(A)}}\left(\because \mathcal{N}(A)^{\perp}=\mathcal{R}\left(A^{*}\right)\right) .
$$

Besides, we derive below two new properties of $A$-adjoint of an operator $T \in \mathcal{B}_{A}(\mathcal{H})$, which are crucial in providing some new proofs of the existing results and in proving new results on $A$-numerical radius inequalities.

$$
T^{\#_{A}} P_{\overline{\mathcal{R}(A)}}=A^{\dagger} T^{*} A A^{\dagger} A=A^{\dagger} T^{*} A=T^{\#_{A}},
$$

and

$$
P_{\overline{\mathcal{R}(A)}} T^{\#_{A}}=A^{\dagger} A A^{\dagger} T^{*} A=A^{\dagger} T^{*} A=T^{\#_{A}} .
$$

An operator $T \in \mathcal{B} \Leftarrow \mathcal{H} \Rightarrow$ is said to be $A$-selfadjoint if $A T$ is selfadjoint, i.e., $A T=T^{*} A$. Observe that if $T$ is $A$-selfadjoint, then $T \in \mathcal{B}_{A}(\mathcal{H})$. However, in general, $T \neq T^{\#_{A}}$. But, $T=T^{\#_{A}}$ if and only if $T$ is $A$-selfadjoint and $\mathcal{R}(T) \subseteq \overline{\mathcal{R}(A)}$. If $T \in \mathcal{B}_{A}(\mathcal{H})$, then $T^{\#_{A}} \in \mathcal{B}_{A}(\mathcal{H}),\left(T^{\#_{A}}\right)^{\#_{A}}=P_{\overline{\mathcal{R}(A)}} T P_{\overline{\mathcal{R}(A)}}$, and $\left(\left(T^{\#_{A}}\right)^{\#_{A}}\right)^{\#_{A}}=T^{\#_{A}}$. Also, $T^{\#_{A}} T$ and $T T^{\#_{A}}$ are $A$-positive operators, and

$$
\left\|T^{\#_{A}} T\right\|_{A}=\left\|T T^{\#_{A}}\right\|_{A}=\|T\|_{A}^{2}=\left\|T^{\#_{A}}\right\|_{A}^{2} .
$$

For any $T_{1}, T_{2} \in \mathcal{B}_{A}(\mathcal{H})$, we have

$$
\begin{aligned}
\left\|T_{1}^{\#_{A}} T_{2}\right\|_{A} & =\sup \left\{\left|\left\langle T_{1}^{\#_{A}} T_{2} x, y\right\rangle\right|: x, y \in \mathcal{H},\|x\|_{A}=\|y\|_{A}=1\right\} \\
& =\sup \left\{\left|\left\langle T_{2} x, T_{1} y\right\rangle\right|: x, y \in \mathcal{H},\|x\|_{A}=\|y\|_{A}=1\right\} \\
& =\sup \left\{\left|\left\langle x, T_{2}^{\#_{A}} T_{1} y\right\rangle\right|: x, y \in \mathcal{H},\|x\|_{A}=\|y\|_{A}=1\right\} \\
& =\sup \left\{\left|\left\langle T_{2}^{\#_{A}} T_{1} y, x\right\rangle\right|: x, y \in \mathcal{H},\|x\|_{A}=\|y\|_{A}=1\right\} \\
& =\left\|T_{2}^{\#_{A}} T_{1}\right\|_{A} .
\end{aligned}
$$

However, the above proof is a very simple one and directly follows from the definition of $A$-norm. An operator $U \in \mathcal{B}_{A}(\mathcal{H})$ is said to be A-unitary if $\|U x\|_{A}=\left\|U^{\#_{A}} x\right\|_{A}=\|x\|_{A}$ for all $x \in \mathcal{H}$. If $T \in \mathcal{B}_{A}(\mathcal{H})$ and $U$ is $A$-unitary, then $w_{A}\left(U^{\#_{A}} T U\right)=w_{A}(T)$. For $T, S \in \mathcal{B}_{A}(\mathcal{H})$, we have $(T S)^{\#_{A}}=S^{\#_{A}} T^{\#_{A}}$, $(T+S)^{\#_{A}}=T^{\#_{A}}+S^{\#_{A}},\|T S\|_{A} \leq\|T\|_{A}\|S\|_{A}$ and $\|T x\|_{A} \leq\|T\|_{A}\|x\|_{A}$ for all $x \in \mathcal{H}$. The real and imaginary part of an operator $T \in \mathcal{B}_{A}(\mathcal{H})$ as $\operatorname{Re}_{A}(T)=\frac{T+T^{\#_{A}}}{2}$ and $\operatorname{Im}_{A}(T)=\frac{T-T^{H_{A}}}{2 i}$. An interested reader may refer [1, 2] for further properties of operators on semi-Hilbertian space. From the definition of $A$-numerical radius of an operator, it follows that

$$
w_{A}(T)=w_{A}\left(T^{\#_{A}}\right) \text { for any } T \in \mathcal{B}_{A}(\mathcal{H}) .
$$

Some interesting results are collected hereunder for further use.

The next result is a combination of [3, Lemma 2.4(i)] and [11, Lemma 2.2].

Lemma 2.1 Let $T_{1}, T_{2}, T_{3}, T_{4} \in \mathcal{B}_{A}(\mathcal{H})$. Then 
(i) $\max \left\{w_{A}\left(T_{1}\right), w_{A}\left(T_{4}\right)\right\}=w_{\text {A }}\left(\left[\begin{array}{cc}T_{1} & O \\ O & T_{4}\end{array}\right]\right) \leq w_{\text {A }}\left(\left[\begin{array}{ll}T_{1} & T_{2} \\ T_{3} & T_{4}\end{array}\right]\right)$.

(ii) $w_{\mathcal{A}}\left(\left[\begin{array}{cc}O & T_{2} \\ T_{3} & O\end{array}\right]\right) \leq w_{\mathbb{A}}\left(\left[\begin{array}{ll}T_{1} & T_{2} \\ T_{3} & T_{4}\end{array}\right]\right)$.

The other parts of [3, Lemma 2.4] assume the condition $A$ is strictly positive. Rout et al. [11] proved the same result for positive $A$, and the same is stated below.

Lemma 2.2 [11, Lemma 2.4]

Let $T_{1}, T_{2} \in \mathcal{B}_{A}(\mathcal{H})$. Then

(i) $w_{\text {A }}\left(\left[\begin{array}{cc}O & T_{1} \\ T_{2} & O\end{array}\right]\right)=w_{\text {A }}\left(\left[\begin{array}{cc}O & T_{2} \\ T_{1} & O\end{array}\right]\right)$.

(ii) $w_{\mathcal{A}}\left(\left[\begin{array}{cc}O & T_{1} \\ e^{i \theta} T_{2} & O\end{array}\right]\right)=w_{\mathcal{A}}\left(\left[\begin{array}{cc}O & T_{1} \\ T_{2} & O\end{array}\right]\right)$ for any $\theta \in \mathbb{R}$.

(iii) $\left.w_{\mathrm{A}}\left(\begin{array}{ll}T_{1} & T_{2} \\ T_{2} & T_{1}\end{array}\right]\right)=\max \left\{w_{A}\left(T_{1}+T_{2}\right), w_{A}\left(T_{1}-T_{2}\right)\right\} . \quad$ In $\quad$ particular, $w_{A}\left(\left[\begin{array}{cc}O & T_{2} \\ T_{2} & O\end{array}\right]\right)=w_{A}\left(T_{2}\right)$.

The next result establishes upper and lower bounds for the $\mathbb{A}$-numerical radius of a particular type of $2 \times 2$ operator matrix that is a generalization of (2).

Lemma 2.3 [11, Theorem 2.6]

Let $T_{1}, T_{2} \in \mathcal{B}_{A}(\mathcal{H})$. Then

$$
\max \left\{w_{A}\left(T_{1}\right), w_{A}\left(T_{2}\right)\right\} \leq w_{\text {A }}\left(\left[\begin{array}{cc}
T_{1} & T_{2} \\
-T_{2} & -T_{1}
\end{array}\right]\right) \leq w_{A}\left(T_{1}\right)+w_{A}\left(T_{2}\right) .
$$

Lemma 2.4 [11, Lemma 2.9]

Let $T_{1}, T_{2} \in \mathcal{B}_{A}(\mathcal{H})$. Then,

$$
w_{\text {A }}\left(\left[\begin{array}{cc}
T_{2} & -T_{1} \\
T_{1} & T_{2}
\end{array}\right]\right)=\max \left\{w_{A}\left(T_{1}+i T_{2}\right), w_{A}\left(T_{1}-i T_{2}\right)\right\} .
$$

\section{Main results}

This section begins with the power inequality for semi-Hilbert space that has been proved by Moslehian et al. [8], which states that for $T \in \mathcal{B} \Leftarrow \mathcal{H} \Rightarrow, w_{A}\left(T^{n}\right) \leq w_{A}^{n}(T)$ for $n \in \mathbb{N}$. Using this, we prove the following theorem. 
Theorem 3.1 Let $T_{1}, T_{2}, T_{3}, T_{4} \in \mathcal{B}_{A}(\mathcal{H})$ and $T=\left[\begin{array}{ll}T_{1} & T_{2} \\ T_{3} & T_{4}\end{array}\right]$. Then

$$
\max \left\{w_{A}^{1 / 2}\left(T_{2} T_{3}\right), w_{A}^{1 / 2}\left(T_{3} T_{2}\right)\right\} \leq w_{A}\left(\left[\begin{array}{cc}
O & T_{2} \\
T_{3} & O
\end{array}\right]\right) .
$$

Proof Here,

$$
w_{\text {A }}\left(\left[\begin{array}{cc}
O & T_{2} \\
T_{3} & O
\end{array}\right]\right) \leq w_{\text {A }}\left(\left[\begin{array}{ll}
T_{1} & T_{2} \\
T_{3} & T_{4}
\end{array}\right]\right) \text { by Lemma } 2.1 \text {. }
$$

Now,

$$
\begin{aligned}
\max \left\{w_{A}\left(T_{2} T_{3}\right), w_{A}\left(T_{3} T_{2}\right)\right\} & =w_{\mathbb{A}}\left(\left[\begin{array}{cc}
T_{2} T_{3} & O \\
O & T_{3} T_{2}
\end{array}\right]\right) \\
& =w_{\mathbb{A}}\left(\left[\begin{array}{cc}
O & T_{2} \\
T_{3} & O
\end{array}\right]\left[\begin{array}{cc}
O & T_{2} \\
T_{3} & O
\end{array}\right]\right) \\
& =w_{\mathbb{A}}\left(\left[\begin{array}{ll}
O & T_{2} \\
T_{3} & O
\end{array}\right]\right) \\
& \leq w_{\mathbb{A}}^{2}\left(\left[\begin{array}{ll}
O & T_{2} \\
T_{3} & O
\end{array}\right]\right) \quad \because w_{A}\left(T^{n}\right) \leq w_{A}^{n}(T)(\text { see }[8]) .
\end{aligned}
$$

This implies

$$
\max \left\{w_{A}^{1 / 2}\left(T_{2} T_{3}\right), w_{A}^{1 / 2}\left(T_{3} T_{2}\right)\right\} \leq w_{A}\left(\left[\begin{array}{cc}
O & T_{2} \\
T_{3} & O
\end{array}\right]\right) .
$$

Remark 3.2 Using Lemma 2.1 and a known inequality $w_{\mathbb{A}}(T) \leq \frac{1}{2}\left(\|T\|_{\mathbb{A}}+\left\|T^{2}\right\|_{\mathbb{A}}^{1 / 2}\right)$, Theorem 3.1 implies that

$$
\max \left\{w_{A}^{1 / 2}\left(T_{2} T_{3}\right), w_{A}^{1 / 2}\left(T_{3} T_{2}\right)\right\} \leq w_{\mathbb{A}}\left(\left[\begin{array}{cc}
O & T_{2} \\
T_{3} & O
\end{array}\right]\right) \leq \frac{1}{2}\left(\|T\|_{\mathbb{A}}+\left\|T^{2}\right\|_{\mathbb{A}}^{1 / 2}\right) .
$$

Example Let $T_{2}=\left[\begin{array}{ll}1 & 0 \\ 0 & 2\end{array}\right], T_{3}=\left[\begin{array}{ll}1 & 0 \\ 0 & 1\end{array}\right] \quad$ and $\quad A=\left[\begin{array}{ll}1 & 0 \\ 0 & 0\end{array}\right] . \quad$ Then, $w_{A}\left(T_{2} T_{3}\right)=w_{A}\left(T_{3} T_{2}\right)=w_{A}\left(\left[\begin{array}{ll}1 & 0 \\ 0 & 2\end{array}\right]\right)=1$.

By Theorem 3.1, we thus have

$$
w_{\mathrm{A}}\left(\left[\begin{array}{cc}
O & T_{2} \\
T_{3} & O
\end{array}\right]\right) \geq 1 .
$$

We generalize some of the results of [6] now. Using Lemma 2.2, one can now state [3, Theorem 3.1] without assuming the condition $A>0$. Its proof takes the 
same steps as in the [3, proof of Theorem 3.1] after the use of Lemma 2.2, and hence the proof is omitted.

Lemma 3.3 Let $T, S, X, Y \in \mathcal{B}_{A}(\mathcal{H})$. Then

$$
w_{A}\left(T X S^{\#_{A}} \pm S Y T^{\#_{A}}\right) \leq 2\|T\|_{A}\|S\|_{A} w_{\text {A }}\left[\begin{array}{ll}
O & X \\
Y & O
\end{array}\right] .
$$

In particular, putting $Y=X$

$$
w_{A}\left(T X S^{\#_{A}} \pm S X T^{\#_{A}}\right) \leq 2\|T\|_{A}\|S\|_{A} w_{A}(X) .
$$

Considering $X=Y=Q$ and $T=I$ in Lemma 3.3, we get the following corollary.

Corollary 3.4 Let $Q, S \in \mathcal{B}_{A}(\mathcal{H})$. Then,

$$
w_{A}\left(Q S^{\#_{A}} \pm S Q\right) \leq 2\|S\|_{A} w_{A}(Q)
$$

It is well known that $P_{\overline{\mathcal{R}(A)}} T \neq T P_{\overline{\mathcal{R}(A)}}$ for $T \in \mathcal{B}_{A}(\mathcal{H})$ (even if $A$ and $T$ are finite matrices). And the equality holds if $\mathcal{N}(A)^{\perp}$ is invariant under $T$. The following result shows that $w_{A}\left(P_{\overline{\mathcal{R}(A)}} T\right)$ and $w_{A}\left(T P_{\overline{\mathcal{R}(A)}}\right)$ are same for all $T \in \mathcal{B}_{A}(\mathcal{H})$ even though $\mathcal{N}(A)^{\perp}$ is not invariant under $T$.

Theorem $3.5 w_{A}\left(P_{\overline{\mathcal{R}(A)}} T\right)=w_{A}\left(T P_{\overline{\mathcal{R}(A)}}\right)=w_{A}(T)$ for any $T \in \mathcal{B}_{A}(\mathcal{H})$.

Proof

$$
\begin{aligned}
w_{A}\left(P_{\overline{\mathcal{R}(A)}} T\right) & =w_{A}\left(\left(P_{\overline{\mathcal{R}(A)}} T\right)^{\#_{A}}\right) & & \left(\because w_{A}(T)=w_{A}\left(T^{\#_{A}}\right)\right) \\
& =w_{A}\left(T^{\#_{A}} P_{\overline{\mathcal{R}(A)}}\right) & & \left(\because(T S)^{\#_{A}}=S^{\#_{A}} T^{\#_{A}} \&\left(P_{\overline{\mathcal{R}(A)}}\right)^{\#_{A}}=P_{\overline{\mathcal{R}(A)}}\right) \\
& =w_{A}\left(T^{\#_{A}}\right) & & \text { by }(4) \\
& =w_{A}(T) . & &
\end{aligned}
$$

Again,

$$
\begin{aligned}
w_{A}\left(T P_{\overline{\mathcal{R}(A)}}\right) & =w_{A}\left(\left(T P_{\overline{\mathcal{R}(A)}}\right)^{\#_{A}}\right) & & \left(\because w_{A}(T)=w_{A}\left(T^{\#_{A}}\right)\right) \\
& =w_{A}\left(P_{\overline{\mathcal{R}(A)}} T^{\#_{A}}\right) & & \left(\because(T S)^{\#_{A}}=S^{\#_{A}} T^{\#_{A}} \&\left(P_{\overline{\mathcal{R}(A)}}\right)^{\#_{A}}=P_{\overline{\mathcal{R}(A)}}\right) \\
& =w_{A}\left(T^{\#_{A}}\right) & & \text { by }(5) \\
& =w_{A}(T) . & &
\end{aligned}
$$

We, therefore, have

$$
w_{A}\left(P_{\overline{\mathcal{R} \Leftarrow \mathcal{A} \Rightarrow}} T\right)=w_{A}\left(T P_{\overline{\mathcal{R} \Leftarrow \mathcal{A} \Rightarrow}}\right)=w_{A}(T) .
$$

The next result provides an estimate for lower bound of $\mathbb{A}$-numerical radius of a $2 \times 2$ operator matrix. 
Theorem 3.6 Let $T_{1}, T_{2}, T_{3}, T_{4} \in \mathcal{B}_{A}(\mathcal{H})$. Then $w_{\mathcal{A}}\left(\left[\begin{array}{ll}T_{1} & T_{2} \\ T_{3} & T_{4}\end{array}\right]\right) \geq \frac{1}{2} \max \{\alpha, \beta\}$, where $\alpha=\max \left\{w_{A}\left(T_{1}+T_{2}+T_{3}+T_{4}\right), w_{A}\left(T_{1}+T_{4}-T_{2}-T_{3}\right)\right\}$ and

$$
\beta=\max \left\{w_{A}\left(T_{1}+T_{4}+i\left(T_{2}-T_{3}\right)\right), w_{A}\left(T_{1}+T_{4}-i\left(T_{2}-T_{3}\right)\right)\right\} .
$$

Proof Let $T=\left[\begin{array}{ll}T_{1}^{\#_{A}} & T_{3}^{\#_{A}} \\ T_{2}^{\#_{A}} & T_{4}^{\#_{A}}\end{array}\right]$ and $Q=\left[\begin{array}{ll}O & I \\ I & O\end{array}\right]$. To show that $Q$ is A-unitary, we need to prove that $\|x\|_{\mathbb{A}}=\|Q x\|_{\mathbb{A}}=\left\|Q^{\#_{\AA}} x\right\|_{\mathbb{A}}$. So,

$$
\begin{aligned}
Q^{\#_{A}} & =\left[\begin{array}{cc}
O & I^{\#_{A}} \\
I^{\#_{A}} & O
\end{array}\right] \quad\left(\because\left[\begin{array}{ll}
T_{1} & T_{2} \\
T_{3} & T_{4}
\end{array}\right]^{\#_{A}}=\left[\begin{array}{cc}
T_{1}^{\#_{A}} & T_{3}^{\#_{A}} \\
T_{2}^{\#_{A}} & T_{4}^{\#_{A}}
\end{array}\right]\right) \\
& =\left[\begin{array}{cc}
O & P_{\overline{\mathcal{R}(A)}} \\
P_{\overline{\mathcal{R}(A)}} & O
\end{array}\right] \quad\left(\because \mathcal{N}(A)^{\perp}=\overline{\mathcal{R}\left(A^{*}\right)} \& \mathcal{R}\left(A^{*}\right)=\mathcal{R}(A)\right) .
\end{aligned}
$$

This in turn implies $Q Q^{\#_{\AA}}=\left[\begin{array}{cc}P_{\overline{\mathcal{R}(A)}} & O \\ O & P_{\overline{\mathcal{R}(A)}}\end{array}\right]=Q^{\#_{\AA}} Q . \quad$ Now, for $x=\left(x_{1}, x_{2}\right) \in \mathcal{H} \bigoplus \mathcal{H}$, we have

$$
\begin{aligned}
\|Q x\|_{\mathbb{A}}^{2}=\langle Q x, Q x\rangle_{\mathbb{A}}=\left\langle Q^{\#_{\mathbb{A}}} Q x, x\right\rangle_{\mathbb{A}} & =\left\langle\left[\begin{array}{cc}
P_{\overline{\mathcal{R}(A)}} & O \\
O & P_{\overline{\mathcal{R}(A)}}
\end{array}\right]\left[\begin{array}{l}
x_{1} \\
x_{2}
\end{array}\right],\left[\begin{array}{l}
x_{1} \\
x_{2}
\end{array}\right]\right\rangle_{\mathbb{A}} \\
& =\left\langle\left[\begin{array}{cc}
A P_{\overline{\mathcal{R}(A)}} & O \\
O & A P_{\overline{\mathcal{R}(A)}}
\end{array}\right]\left[\begin{array}{l}
x_{1} \\
x_{2}
\end{array}\right],\left[\begin{array}{l}
x_{1} \\
x_{2}
\end{array}\right]\right\rangle \\
& =\left\langle\left[\begin{array}{cc}
A A^{\dagger} A & O \\
O & A A^{\dagger} A
\end{array}\right]\left[\begin{array}{l}
x_{1} \\
x_{2}
\end{array}\right],\left[\begin{array}{l}
x_{1} \\
x_{2}
\end{array}\right]\right\rangle \\
& =\left\langle\left[\begin{array}{ll}
A & O \\
O & A
\end{array}\right]\left[\begin{array}{l}
x_{1} \\
x_{2}
\end{array}\right],\left[\begin{array}{l}
x_{1} \\
x_{2}
\end{array}\right]\right\rangle \\
& =\|x\|_{\mathbb{A}}^{2} .
\end{aligned}
$$

So, $\|Q x\|_{\mathbb{A}}=\|x\|_{\mathbb{A}}$. Similarly, it can be proved that $\left\|Q^{\#_{\AA}} x\right\|_{\mathbb{A}}=\|x\|_{\mathbb{A}}$. Thus, $Q$ is an $\mathbb{A}$ -unitary operator. By Corollary 3.4, we obtain

$$
w_{A}\left(T Q^{\#_{A}} \pm Q T\right) \leq 2 w_{\AA}(T) .
$$

So, 


$$
\begin{aligned}
& 2 w_{\mathcal{A}}(T) \geq w_{\mathcal{A}}\left(\left[\begin{array}{cc}
T_{1}^{\#_{A}} & T_{3}^{\#_{A}} \\
T_{2}^{\#_{A}} & T_{4}^{\#_{A}}
\end{array}\right]\left[\begin{array}{cc}
O & P_{\overline{\mathcal{R}(A)}} \\
P_{\overline{\mathcal{R}(A)}} & O
\end{array}\right]+\left[\begin{array}{ll}
O & I \\
I & O
\end{array}\right]\left[\begin{array}{cc}
T_{1}^{\#_{A}} & T_{3}^{\#_{A}} \\
T_{2}^{\#_{A}} & T_{4}^{\#_{A}}
\end{array}\right]\right) \\
& =w_{\text {A }}\left(\left[\begin{array}{ll}
T_{3}^{\#_{A}} P_{\overline{\mathcal{R}(A)}} & T_{1}^{\#_{A}} P_{\overline{\mathcal{R}(A)}} \\
T_{4}^{\#_{A}} P_{\overline{\mathcal{R}(A)}} & T_{2}^{\#_{A}} P_{\overline{\mathcal{R}(A)}}
\end{array}\right]+\left[\begin{array}{ll}
T_{2}^{\#_{A}} & T_{4}^{\#_{A}} \\
T_{1}^{\#_{A}} & T_{3}^{\#_{A}}
\end{array}\right]\right) \\
& =w_{\text {A }}\left(\left[\begin{array}{cc}
T_{3}^{\#_{A}} & T_{1}^{\#_{A}} \\
T_{4}^{\#_{A}} & T_{2}^{\#_{A}}
\end{array}\right]+\left[\begin{array}{cc}
T_{2}^{\#_{A}} & T_{4}^{\#_{A}} \\
T_{1}^{\#_{A}} & T_{3}^{\#_{A}}
\end{array}\right]\right) \quad \text { by (4) } \\
& =w_{\mathrm{A}}\left(\left[\begin{array}{cc}
T_{3}^{\#_{A}}+T_{2}^{\#_{A}} & T_{1}^{\#_{A}}+T_{4}^{\#_{A}} \\
T_{4}^{\#_{A}}+T_{1}^{\#_{A}} & T_{2}^{\#_{A}}+T_{3}^{\#_{A}}
\end{array}\right]\right) \\
& =w_{\mathrm{A}}\left(\left[\begin{array}{ll}
T_{2}+T_{3} & T_{4}+T_{1} \\
T_{4}+T_{1} & T_{2}+T_{3}
\end{array}\right]^{\#_{\AA}}\right) \\
& =w_{\text {A }}\left(\left[\begin{array}{ll}
T_{2}+T_{3} & T_{4}+T_{1} \\
T_{4}+T_{1} & T_{2}+T_{3}
\end{array}\right]\right) \text {. }
\end{aligned}
$$

Hence, we have

$$
2 w_{\mathrm{A}}\left(\left[\begin{array}{ll}
T_{1} & T_{2} \\
T_{3} & T_{4}
\end{array}\right]\right)=2 w_{\mathrm{A}}\left(\left[\begin{array}{ll}
T_{1}^{\#_{A}} & T_{3}^{\#_{A}} \\
T_{2}^{\#_{A}} & T_{4}^{\#_{A}}
\end{array}\right]\right) \geq w_{\text {A }}\left(\left[\begin{array}{ll}
T_{2}+T_{3} & T_{4}+T_{1} \\
T_{4}+T_{1} & T_{2}+T_{3}
\end{array}\right]\right) .
$$

By (13) and Lemma 2.2, we obtain

$$
w_{\text {A }}\left(\left[\begin{array}{ll}
T_{1} & T_{2} \\
T_{3} & T_{4}
\end{array}\right]\right) \geq \frac{1}{2} \max \left\{w_{A}\left(T_{1}+T_{2}+T_{3}+T_{4}\right), w_{A}\left(T_{2}+T_{3}-T_{4}-T_{1}\right)\right\} .
$$

Again, applying Corollary 3.4 and taking $T=\left[\begin{array}{cc}T_{1}^{\#_{A}} & T_{3}^{\#_{A}} \\ T_{2}^{\#_{A}} & T_{4}^{\#_{A}}\end{array}\right]$ and $Q=\left[\begin{array}{cc}O & I \\ -I & O\end{array}\right]$, it can be shown that $Q$ is A-unitary and $w_{\mathcal{A}}\left(T Q^{\#_{A}} \pm Q T\right) \leq 2 w_{\AA}(T)$. Therefore,

$$
\begin{aligned}
2 w_{\mathcal{A}}(T) & \geq w_{\text {A }}\left(\left[\begin{array}{ll}
T_{1}^{\#_{A}} & T_{3}^{\#_{A}} \\
T_{2}^{\#_{A}} & T_{4}^{\#_{A}}
\end{array}\right]\left[\begin{array}{cc}
O & -P_{\overline{\mathcal{R}(A)}} \\
P_{\overline{\mathcal{R}(A)}} & O
\end{array}\right]-\left[\begin{array}{cc}
O & I \\
-I & O
\end{array}\right]\left[\begin{array}{ll}
T_{1}^{\#_{A}} & T_{3}^{\#_{A}} \\
T_{2}^{\#_{A}} & T_{4}^{\#_{A}}
\end{array}\right]\right) \\
& =w_{\text {A }}\left(\left[\begin{array}{ll}
T_{3}^{\#_{A}} P_{\overline{\mathcal{R}(A)}} & -T_{1}^{\#_{A}} P_{\overline{\mathcal{R}(A)}} \\
T_{4}^{\#_{A}} P_{\overline{\mathcal{R}(A)}} & -T_{2}^{\#_{A}} P_{\overline{\mathcal{R}(A)}}
\end{array}\right]-\left[\begin{array}{cc}
T_{2}^{\#_{A}} & T_{4}^{\#_{A}} \\
-T_{1}^{\#_{A}} & -T_{3}^{\#_{A}}
\end{array}\right]\right) \\
& =w_{\text {A }}\left(\left[\begin{array}{ll}
-T_{2}^{\#_{A}}+T_{3}^{\#_{A}} & -T_{4}^{\#_{A}}-T_{1}^{\#_{A}} \\
T_{4}^{\#_{A}}+T_{1}^{\#_{A}} & -T_{2}^{\#_{A}}+T_{3}^{\#_{A}}
\end{array}\right]\right) \text { by (4) } \\
& =w_{\text {A }}\left(\left[\begin{array}{ll}
-T_{2}+T_{3} & T_{4}+T_{1} \\
-T_{4}-T_{1} & -T_{2}+T_{3}
\end{array}\right]\right) .
\end{aligned}
$$

By Lemma 2.4, we, therefore, achieve the following: 


$$
w_{\text {A }}\left(\left[\begin{array}{ll}
T_{1} & T_{2} \\
T_{3} & T_{4}
\end{array}\right]\right) \geq \frac{1}{2} \max \left\{w_{A}\left(T_{4}+T_{1}-i\left(T_{2}-T_{3}\right)\right), w_{A}\left(T_{4}+T_{1}+i\left(T_{2}-T_{3}\right)\right)\right\} .
$$

From (14) and (15), we get the desired result.

Example Let $T_{1}=T_{2}=T_{3}=T_{4}=\left[\begin{array}{ll}1 & 0 \\ 0 & 2\end{array}\right]$ and $A=\left[\begin{array}{ll}1 & 0 \\ 0 & 0\end{array}\right]$. Then $w_{A}\left(\left[\begin{array}{ll}1 & 0 \\ 0 & 2\end{array}\right]\right)=1$.

By Theorem 3.6, we have $\alpha=4, \beta=2$ and

$$
w_{\text {A }}\left(\left[\begin{array}{ll}
T_{1} & T_{2} \\
T_{3} & T_{4}
\end{array}\right]\right) \geq 2 .
$$

Remark 3.7 Using [11, Theorem 2.1] in the above example, it is easy to find that

$$
w_{\text {A }}\left(\left[\begin{array}{ll}
T_{1} & T_{2} \\
T_{3} & T_{4}
\end{array}\right]\right) \leq 2 .
$$

So, we have

$$
w_{\mathrm{A}}\left(\left[\begin{array}{ll}
T_{1} & T_{2} \\
T_{3} & T_{4}
\end{array}\right]\right)=2 .
$$

Remark 3.8 Using [11, Theorem 2.15] in the above example, it is easy to find

$$
w_{\text {A }}\left(\left[\begin{array}{ll}
T_{1} & T_{2} \\
T_{3} & T_{4}
\end{array}\right]\right) \geq 1 .
$$

So, Theorem 3.6 is a refinement of Theorem 2.15 of [11].

As a special case of the above result, we obtain a corollary in Krein space setting. Let $J$ be a non-scalar Hermitian involution operator in $\mathcal{B}(\mathbb{W})$, where $\mathbb{W}$ is a separable Hilbert space. Then, space $\left(\mathbb{H},\langle., .\rangle_{J}\right)$ is called Krein space [4]. Note that the $J$-adjoint operator of $T \in \mathcal{B}(\mathbb{M})$ is the unique operator in $\mathcal{B}(\mathbb{M})$ such that $\langle T x, y\rangle_{J}=\left\langle x, T^{\#_{J}} y\right\rangle_{J}$, for all $x, y \in \mathbb{H}$. Therefore, we have $T^{\#_{J}}=J T^{*} J$ and $\left(T^{\#_{J}}\right)^{\#_{J}}=T$. One can observe that Lemmas 2.1 and 2.2 hold in the setting of Krein spaces for $T_{1}, T_{2} \in \mathcal{B}(\mathbb{W})$. We provide below a lemma for the $J$-adjoint operator of an $n \times n$ operator matrix.

$\begin{aligned} \text { Lemma } 3.9 \text { Let } T= & {\left[\begin{array}{cccc}T_{11} & T_{12} & \cdots & T_{1 n} \\ T_{21} & T_{22} & \cdots & T_{2 n} \\ \vdots & \vdots & \cdots & \vdots \\ T_{n 1} & T_{n 2} & \cdots & T_{n n}\end{array}\right] \text {, where } T_{i j} \in \mathcal{B}(\mathbb{W}) \text {. Then } } \\ & {\left[\begin{array}{cccc}T_{11} & T_{12} & \cdots & T_{1 n} \\ T_{21} & T_{22} & \cdots & T_{2 n} \\ \vdots & \vdots & \cdots & \vdots \\ T_{n 1} & T_{n 2} & \cdots & T_{n n}\end{array}\right]=\left[\begin{array}{cccc}T_{11}^{\#_{J}} & T_{21}^{\#_{J}} & \cdots & T_{n_{J}}^{\#_{J}} \\ T_{12}^{\#_{J}} & T_{22}^{\#_{J}} & \cdots & T_{n 2}^{\#_{J}} \\ \vdots & \vdots & \cdots & \vdots \\ T_{1 n}^{\#_{J}} & T_{2 n}^{\#_{J}} & \cdots & T_{n n}^{\#_{J}}\end{array}\right] . }\end{aligned}$ 


$$
\begin{aligned}
& {\left[\begin{array}{cccc}
T_{11} & T_{12} & \cdots & T_{1 n} \\
T_{21} & T_{22} & \cdots & T_{2 n}^{\#_{J}} \\
\vdots & \vdots & \cdots & \vdots \\
T_{n 1} & T_{n 2} & \cdots & T_{n n}
\end{array}\right]^{-}} \\
& =\left[\begin{array}{cccc}
J & O & \cdots & O \\
O & J & \cdots & O \\
\vdots & \vdots & \cdots & \vdots \\
O & O & \cdots & J
\end{array}\right]\left[\begin{array}{cccc}
T_{11} & T_{12} & \cdots & T_{1 n} \\
T_{21} & T_{22} & \cdots & T_{2 n} \\
\vdots & \vdots & \cdots & \vdots \\
T_{n 1} & T_{n 2} & \cdots & T_{n n}
\end{array}\right]^{*}\left[\begin{array}{cccc}
J & O & \cdots & O \\
O & J & \cdots & O \\
\vdots & \vdots & \cdots & \vdots \\
O & O & \cdots & J
\end{array}\right] \\
& =\left[\begin{array}{cccc}
J & O & \cdots & O \\
O & J & \cdots & O \\
\vdots & \vdots & \cdots & \vdots \\
O & O & \cdots & J
\end{array}\right]\left[\begin{array}{cccc}
T_{11}^{*} & T_{21}^{*} & \cdots & T_{n 1}^{*} \\
T_{12}^{*} & T_{22}^{*} & \cdots & T_{n 2}^{*} \\
\vdots & \vdots & \cdots & \vdots \\
T_{1 n}^{*} & T_{2 n}^{*} & \cdots & T_{n n}^{*}
\end{array}\right]\left[\begin{array}{cccc}
J & O & \cdots & O \\
O & J & \cdots & O \\
\vdots & \vdots & \cdots & \vdots \\
O & O & \cdots & J
\end{array}\right] \\
& =\left[\begin{array}{cccc}
J T_{11}^{*} J & J T_{21}^{*} J & \cdots & J T_{n 1}^{*} J \\
J T_{12}^{*} J & J T_{22}^{*} J & \cdots & J T_{n 2}^{*} J \\
\vdots & \vdots & \cdots & \vdots \\
J T_{1 n}^{*} J & J T_{2 n}^{*} J & \cdots & J T_{n n}^{*} J
\end{array}\right] \\
& =\left[\begin{array}{cccc}
T_{11}^{\#_{J}} & T_{21}^{\#_{J}} & \cdots & T_{n^{1}}^{\#_{J}} \\
T_{12}^{\#_{J}} & T_{22}^{\#_{J}} & \cdots & T_{n 2}^{\#_{J}} \\
\vdots & \vdots & \cdots & \vdots \\
T_{1 n}^{\#_{J}} & T_{2 n}^{\#_{J}} & \cdots & T_{n n}^{\#_{J}}
\end{array}\right] .
\end{aligned}
$$

Proof

We state below another lemma for Krein space operators whose proof follows from [3, Theorem 3.1].

Lemma 3.10 Let $T, S, X, Y \in \mathcal{B}(\mathbb{H})$. Then,

$$
w_{J}\left(T X S^{\#_{J}} \pm S Y T^{\#}\right) \leq 2\|T\|_{J}\|S\|_{J} w_{J}\left(\left[\begin{array}{cc}
O & X \\
Y & O
\end{array}\right]\right) .
$$

In particular, if $X=Y$, then

$$
w_{J}\left(T X S^{\#_{J}} \pm S X T^{\#_{J}}\right) \leq 2\|T\|_{J}\|S\|_{J} w_{J}(X),
$$

and if $X=Y=Q, T=I$, then

$$
w_{J}\left(Q S^{\#_{J}} \pm S Q\right) \leq 2\|S\|_{J} w_{J}(Q) .
$$

Based on these results, we have the following corollary of Theorem 3.6.

Corollary 3.11 Let $T_{1}, T_{2}, T_{3}, T_{4} \in \mathcal{B}(\mathbb{W})$. Then $w_{\rrbracket}\left(\left[\begin{array}{ll}T_{1} & T_{2} \\ T_{3} & T_{4}\end{array}\right]\right) \geq \frac{1}{2} \max \{\alpha, \beta\}$, where $\alpha=\max \left\{w_{J}\left(T_{1}+T_{2}+T_{3}+T_{4}\right), w_{J}\left(T_{1}+T_{4}-T_{2}-T_{3}\right)\right\}$ and $\beta=\max \left\{w_{J}\left(T_{1}+T_{4}+i\left(T_{2}-T_{3}\right)\right), w_{J}\left(T_{1}+T_{4}-i\left(T_{2}-T_{4}\right)\right)\right\}$. 
We provide below a lower bound for $\mathbb{A}$-numerical radius inequality of an operator matrix.

Theorem 3.12 Let $T_{1}, T_{2} \in \mathcal{B}_{A}(\mathcal{H})$. Then,

$$
w_{\text {A }}\left(\left[\begin{array}{ll}
T_{1} & T_{2} \\
O & O
\end{array}\right]\right) \geq \frac{1}{2} \max \left\{w_{A}\left(T_{1}+i T_{2}\right), w_{A}\left(T_{1}-i T_{2}\right)\right\} .
$$

Proof Suppose that $T=\left[\begin{array}{ll}T_{1}^{\#_{A}} & O \\ T_{2}^{\#_{A}} & O\end{array}\right]$ and $Q=\left[\begin{array}{cc}O & -I \\ I & O\end{array}\right]$. It then follows that $Q$ is $\mathbb{A}$-unitary. So, $\|Q\|_{\mathbb{A}}=1$. Using Corollary 3.4, we get

$$
2 w_{\text {A }}(T) \geq w_{\text {A }}\left(T Q^{\#_{A}}-Q T\right) .
$$

Now,

$$
\begin{aligned}
& w_{\text {A }}(T) \geq \frac{1}{2} w_{\text {A }}\left(T Q^{\#_{\AA}}-Q T\right) \\
& =\frac{1}{2} w_{\mathcal{A}}\left(\left[\begin{array}{cc}
T_{1}^{\#_{A}} & O \\
T_{2}^{\#_{A}} & O
\end{array}\right]\left[\begin{array}{cc}
O & P_{\overline{\mathcal{R}(A)}} \\
-P_{\overline{\mathcal{R}(A)}} & O
\end{array}\right]-\left[\begin{array}{cc}
O & -I \\
I & O
\end{array}\right]\left[\begin{array}{cc}
T_{1}^{\#_{A}} & O \\
T_{2}^{\#_{A}} & O
\end{array}\right]\right) \\
& =\frac{1}{2} w_{\mathcal{A}}\left(\left[\begin{array}{ll}
O & T_{1}^{\#_{A}} P_{\overline{\mathcal{R}(A)}} \\
O & T_{2}^{\#_{A}} P_{\overline{\mathcal{R}(A)}}
\end{array}\right]-\left[\begin{array}{cc}
-T_{2}^{\#_{A}} & O \\
T_{1}^{\#_{A}} & O
\end{array}\right]\right) \\
& =\frac{1}{2} w_{\text {A }}\left(\left[\begin{array}{cc}
T_{2}^{\#_{A}} & T_{1}^{\#_{A}} \\
-T_{1}^{\#_{A}} & T_{2}^{\#_{A}}
\end{array}\right]\right) b y(4) \\
& =\frac{1}{2} w_{\mathbb{A}}\left(\left[\begin{array}{cc}
T_{2} & -T_{1} \\
T_{1} & T_{2}
\end{array}\right]^{\#_{\mathbb{A}}}\right) \\
& =\frac{1}{2} w_{\text {A }}\left(\left[\begin{array}{cc}
T_{2} & -T_{1} \\
T_{1} & T_{2}
\end{array}\right]\right) \text {. }
\end{aligned}
$$

By Lemma 2.4, we thus have

$$
w_{\text {A }}\left(\left[\begin{array}{cc}
T_{1} & T_{2} \\
O & O
\end{array}\right]\right)=w_{\text {A }}\left(\left[\begin{array}{ll}
T_{1}^{\#_{A}} & O \\
T_{2}^{\#_{A}} & O
\end{array}\right]\right) \geq \frac{1}{2} \max \left\{w_{\text {A }}\left(T_{1}+i T_{2}\right), w_{\text {A }}\left(T_{1}-i T_{2}\right)\right\} .
$$

Remark 3.13 One can observe that Theorem 3.12 can also be proved by taking $T_{3}=T_{4}=O$ in Theorem 3.6, and is shown below.

By Theorem 3.6, we have 


$$
\begin{aligned}
w_{\text {A }}\left(\left[\begin{array}{cc}
T_{1} & T_{2} \\
O & O
\end{array}\right]\right) & \geq \frac{1}{2} \max \left\{w_{A}\left(T_{1}+T_{2}\right), w_{A}\left(T_{1}-T_{2}\right), w_{A}\left(T_{1}+i T_{2}\right), w_{A}\left(T_{1}-i T_{2}\right)\right\} \\
& \geq \frac{1}{2} \max \left\{w_{A}\left(T_{1}+i T_{2}\right), w_{A}\left(T_{1}-i T_{2}\right)\right\} .
\end{aligned}
$$

Corollary 3.14 Let $T=P+i Q$ be the cartesian decomposition in $\mathcal{B}_{A}(\mathcal{H})$. Then

\section{Proof}

$$
\begin{aligned}
\frac{1}{2} w_{A}(T) \leq \min & \left\{w_{\mathrm{A}}\left(\left[\begin{array}{ll}
P & Q \\
O & O
\end{array}\right]\right), w_{\mathrm{A}}\left(\left[\begin{array}{ll}
O & P \\
Q & O
\end{array}\right]\right)\right\} . \\
w_{\mathrm{A}}\left(\left[\begin{array}{ll}
P & Q \\
O & O
\end{array}\right]\right) & \geq \frac{1}{2} \max \left\{w_{A}(P+i Q), w_{A}(P-i Q)\right\} \\
& =\frac{1}{2} \max \left\{w_{A}(T), w_{A}\left(T^{\#_{A}}\right)\right\} \\
& =\frac{1}{2} w_{A}(T) .
\end{aligned}
$$

Using Lemma 2.2, we obtain

$$
w_{\text {A }}\left(\left[\begin{array}{ll}
O & P \\
Q & O
\end{array}\right]\right)=w_{\mathrm{A}}\left(\left[\begin{array}{cc}
O & P \\
i Q & O
\end{array}\right]\right) \geq \frac{1}{2} w_{A}(P \pm i Q)=\frac{1}{2} w_{A}(T) .
$$

From (16) and (17), we have

$$
\frac{1}{2} w_{A}(T) \leq \min \left\{w_{\mathrm{A}}\left(\left[\begin{array}{ll}
P & Q \\
O & O
\end{array}\right]\right), w_{\mathrm{A}}\left(\left[\begin{array}{ll}
O & P \\
Q & O
\end{array}\right]\right)\right\} .
$$

One can easily prove the next lemma proceeding as in the proof of [11, Lemma 2.9], and is stated below.

Lemma 3.15 Let $T_{1}, T_{2} \in \mathcal{B}_{J}(\mathbb{M})$. Then

$$
w_{\triangleleft}\left(\left[\begin{array}{cc}
T_{2} & -T_{1} \\
T_{1} & T_{2}
\end{array}\right]\right)=\max \left\{w_{J}\left(T_{1}+i T_{2}\right), w_{J}\left(T_{1}-i T_{2}\right)\right\} .
$$

The next corollary follows from the above lemma.

Corollary 3.16 Let $T_{1}, T_{2} \in \mathcal{B}_{J}(\mathbb{H})$. Then

$$
w_{\triangleright}\left(\left[\begin{array}{ll}
T_{1} & T_{2} \\
O & O
\end{array}\right]\right) \geq \frac{1}{2} \max \left\{w_{J}\left(T_{1}+i T_{2}\right), w_{J}\left(T_{1}-i T_{2}\right)\right\} .
$$

We conclude this section with the following two results for $n \times n$ operator matrices. First, we demonstrate an interesting property of A-numerical radius of an $n \times n$ operator matrix which is a generalization of [10, Lemma 2.1]. 
Theorem 3.17 Let $T=\left[\begin{array}{cccc}T_{11} & T_{12} & \cdots & T_{1 n} \\ T_{21} & T_{22} & \cdots & T_{2 n} \\ \vdots & \vdots & \vdots & \vdots \\ T_{n 1} & T_{n 2} & \cdots & T_{n n}\end{array}\right]$, where $T_{i j} \in \mathcal{B}_{A}(\mathcal{H})$ for $1 \leq i, j \leq n$. Then

$$
w_{\text {A }}\left(\left[\begin{array}{cccc}
T_{11} & O & \cdots & O \\
O & T_{22} & \cdots & O \\
\vdots & \vdots & \vdots & \vdots \\
O & O & \cdots & T_{n n}
\end{array}\right]\right) \leq w_{\text {A }}(T) .
$$

Proof Let $z=e^{\frac{2 \pi i}{n}}$ and $U=\left[\begin{array}{cccc}I & O & \cdots & O \\ O & z I & \cdots & O \\ \vdots & \vdots & \vdots & \vdots \\ O & O & \cdots & z^{n-1} I\end{array}\right]$. It is easy to see that $\bar{z}=z^{-1}=z^{n-1}$ and $|z|=1$. To show that $U$ is $\mathbb{A}$-unitary, we need to prove that $\|x\|_{\mathbb{A}}=\|U x\|_{\mathbb{A}}=\left\|U^{\#_{\mathbb{A}}} x\right\|_{\mathbb{A}}$, for $x=\left(x_{1}, x_{2}, \cdots, x_{n}\right) \in \bigoplus_{i=1}^{n} \mathcal{H}$. Here,

$$
\begin{aligned}
& U^{\#_{\mathbb{A}}}=\left[\begin{array}{cccc}
I & O & \cdots & O \\
O & z I & \cdots & O \\
\vdots & \vdots & \vdots & \vdots \\
O & O & \cdots & z^{n-1} I
\end{array}\right]^{\#_{A}} \\
& =\left[\begin{array}{cccc}
I^{\#_{A}} & O & \cdots & O \\
O & \bar{z} I^{\#_{A}} & \cdots & O \\
\vdots & \vdots & \vdots & \vdots \\
O & O & \cdots & \bar{z}^{n-1} I^{\#_{A}}
\end{array}\right] \\
& =\left[\begin{array}{cccc}
P_{\overline{\mathcal{R}(A)}} & O & \cdots & O \\
O & \bar{z} P_{\overline{\mathcal{R}(A)}} & \cdots & O \\
\vdots & \vdots & \vdots & \vdots \\
O & O & \cdots & \bar{z}^{n-1} P_{\overline{\mathcal{R}(A)}}
\end{array}\right] .
\end{aligned}
$$

This in turn implies $U U^{\#_{A}}=\left[\begin{array}{cccc}P_{\overline{\mathcal{R}}(A)} & O & \cdots & O \\ O & P_{\overline{\mathcal{R}(A)}} & \cdots & O \\ \vdots & \vdots & \vdots & \vdots \\ O & O & \cdots & P_{\overline{\mathcal{R}(A)}}\end{array}\right]=U^{\#_{A}} U$.

Now, for $x=\left(x_{1}, x_{2}, \cdots, x_{n}\right) \in \bigoplus_{i=1}^{n} \mathcal{H}$, we have

$$
\|U x\|_{\mathbb{A}}^{2}=\langle U x, U x\rangle_{\mathbb{A}}=\left\langle U^{\#_{\mathbb{A}}} U x, x\right\rangle_{\mathbb{A}}=\|x\|_{\mathbb{A}}^{2} .
$$

So, $\|U x\|_{\mathbb{A}}=\|x\|_{\mathbb{A}}$. Similarly, $\left\|U^{\#_{\mathbb{A}}} x\right\|_{\mathbb{A}}=\|x\|_{\mathbb{A}}$. Thus, $U$ is an $\mathbb{A}$-unitary operator. Further, a simple calculation shows that 


$$
\left[\begin{array}{cccc}
T_{11}^{\#_{A}} & O & \cdots & O \\
O & T_{22}^{\#_{A}} & \cdots & O \\
\vdots & \vdots & \vdots & \vdots \\
O & O & \cdots & T_{n n}^{\#_{A}}
\end{array}\right]=\frac{1}{n} \sum_{k=0}^{n-1} U^{\#_{A} k} T^{\#_{A}} U^{k}
$$

Therefore,

$$
\begin{aligned}
w_{\mathrm{A}}\left(\left[\begin{array}{cccc}
T_{11}^{\#_{A}} & O & \cdots & O \\
O & T_{22}^{\#_{A}} & \cdots & O \\
\vdots & \vdots & \vdots & \vdots \\
O & O & \cdots & T_{n n}^{\#_{A}}
\end{array}\right]\right) \leq \frac{1}{n} \sum_{k=0}^{n-1} w_{\AA}\left(U^{\#_{\mathrm{A}}} T^{\#_{\AA}} U^{k}\right) \\
\quad=\frac{1}{n} \sum_{k=0}^{n-1} w_{\AA}\left(T^{\#_{\mathrm{A}}}\right) \\
\quad=\frac{1}{n} \sum_{k=0}^{n-1} w_{\AA}(T) \\
=w_{\mathrm{A}}(T) .
\end{aligned}
$$

This implies that

$$
w_{\mathrm{A}}\left(\left[\begin{array}{cccc}
T_{11} & O & \cdots & O \\
O & T_{22} & \cdots & O \\
\vdots & \vdots & \vdots & \vdots \\
O & O & \cdots & T_{n n}
\end{array}\right]^{\#_{\mathrm{A}}}\right)=w_{\mathrm{A}}\left(\left[\begin{array}{cccc}
T_{11} & O & \cdots & O \\
O & T_{22} & \cdots & O \\
\vdots & \vdots & \vdots & \vdots \\
O & O & \cdots & T_{n n}
\end{array}\right]\right) \leq w_{\mathrm{A}}(T) .
$$

The last result provides a relation between A-numerical radius of two diagonal operator matrices, where $\operatorname{diag}\left(T_{1}, \ldots, T_{n}\right)$ means an $n \times n$ diagonal operator matrix with entries $T_{1}, \ldots, T_{n}$.

Theorem 3.18 Let $T_{i} \in \mathcal{B}_{A}(\mathcal{H})$ for $1 \leq i \leq n$. Then

$$
w_{\text {A }}\left(\operatorname{diag}\left(\sum_{i=1}^{n} T_{i}, \ldots, \sum_{i=1}^{n} T_{i}\right)\right) \leq n w_{\mathbb{A}}\left(\operatorname{diag}\left(T_{1}, \ldots, T_{n}\right)\right) .
$$

Proof Here, 


$$
\begin{aligned}
w_{\text {A }}\left(\operatorname{diag}\left(\sum_{i=1}^{n} T_{i}, \ldots, \sum_{i=1}^{n} T_{i}\right)\right) & =w_{A}\left(\sum_{i=1}^{n} T_{i}\right) \text { by Lemma } 2.1 \\
& \leq \sum_{i=1}^{n} w_{A}\left(T_{i}\right) \\
& \leq n \max \left\{w_{A}\left(T_{i}\right): 1 \leq i \leq n\right\} \\
& =n w_{\text {A }}\left(\operatorname{diag}\left(T_{1}, \ldots, T_{n}\right)\right) .
\end{aligned}
$$

\section{Concluding remarks}

In this paper, we have further studied $A$-numerical radius inequalities for operators on semi-Hilbertian space and numerical radius inequalities for operators in Krein space. The important findings are summarized as follows:

- Several upper and lower bounds for a $2 \times 2$ operator matrices are demonstrated.

- Some existing A-numerical radius inequalities are provided by relaxing sufficient condition $A>0$. This shows the importance of the theory of the Moore-Penrose inverse in this field of research.

- Some numerical radius inequalities for operator matrices in Krein space setting are shown as corollaries to our main results.

This paper ends with the note that further work on A-numerical radius for $n \times n$ operator matrices and numerical radius inequalities for operators in Krein space can be studied.

Acknowledgements We would like to express our sincere thanks and gratitude to the editor and the anonymous referees for their valuable comments and suggestions in the improvement of the manuscript. We thank the Government of India for introducing the work from home initiative during the COVID-19 pandemic.

\section{References}

1. Arias, M.L., Corach, G., Gonzalez, M.C.: Metric properties of projections in semi-Hilbertian spaces. Integal Equ. Oper. Theory 62, 11-28 (2008)

2. Arias, M.L., Corach, G., Gonzalez, M.C.: Partial isometries in semi-Hilbertian spaces. Linear Algebra Appl. 428, 1460-1475 (2008)

3. Bhunia, P., Paul, K., Nayak, R.K.: On inequalities for A-numerical radius of operators. Electron. J. Linear Algebra 36, 143-157 (2020)

4. Bognar, J.: Indefinite Inner Product Spaces. Springer, Berlin (1974)

5. Douglas, R.G.: On majorization, factorization, and range inclusion of operators on Hilbert space. Proc. Am. Math. Soc. 17, 413-415 (1966)

6. Hirzallah, O., Kittaneh, F., Shebrawi, K.: Numerical radius inequalities for commutators of Hilbert space operators. Numer. Funct. Anal. Optim. 32, 739-749 (2011) 
7. Moslehian, M.S., Kian, M., Xu, Q.: Positivity of $2 \times 2$ block matrices of operators. Banach J. Math. Anal. 13, 726-743 (2019)

8. Moslehian, M.S., Xu, Q., Zamani, A.: Seminorm and numerical radius inequalities of operators in semi-Hilbertian spaces. Linear Algebra Appl. 591, 299-321 (2020)

9. Nashed, M.Z.: Generalized Inverses and Applications. Academic Press, New York (1976)

10. Omidvar, M.E., Moslehian, M.S., Niknam, A.: Some numerical radius inequalities for Hilbert space operators. Involve 2, 471-478 (2009)

11. Rout, N.C., Sahoo, S., Mishra, D.: On A-numerical radius inequalities for $2 \times 2$ operator matrices. Linear Multilinear Algebra (2020). https://doi.org/10.1080/03081087.2020.1810201

12. Rout, N.C., Sahoo, S., Mishra, D.: Some A-numerical radius inequalities for semi-Hilbertian space operators. Linear Multilinear Algebra 69, 980-996 (2021)

13. Saddi, A.: A-normal operators in semi Hilbertian spaces. Aust. J. Math. Anal. Appl. 9, 1-12 (2012)

14. Xu, Q., Ye, Z., Zamani, A.: Some upper bounds for the A-numerical radius of $2 \times 2$ block matrices. Adv. Oper. Theory (2021). https://doi.org/10.1007/s43036-020-00102-5

15. Zamani, A.: A-numerical radius and product of semi-Hilbertian operators. Bull. Iran. Math. Soc. 47, 371-377 (2021)

16. Zamani, A.: A-Numerical radius inequalities for semi-Hilbertian space operators. Linear Algebra Appl. 578, 159-183 (2019) 